Article

\title{
Analysis of Stability-To-Chaos in the Dynamic Evolution of Network Traffic Flows under a Dual Updating Mechanism
}

\author{
Shixu Liu ${ }^{1, * \mathbb{C}}$, Hao Yan ${ }^{1}$, Said M. Easa ${ }^{2}{ }^{\oplus}$, Lidan Guo $^{1}$ and Yingnuo Tang ${ }^{1}$ \\ 1 College of Civil Engineering, Fuzhou University, Fuzhou 350108, China; N160520091@fzu.edu.cn (H.Y.); \\ N170520088@fzu.edu.cn (L.G.); N170527158@fzu.edu.cn (Y.T.) \\ 2 Department of Civil Engineering, Ryerson University, Toronto, ON M5B 2K3, Canada; seasa@ryerson.ca \\ * Correspondence: liushixu@fzu.edu.cn
}

Received: 4 October 2018; Accepted: 9 November 2018; Published: 13 November 2018

check for updates

\begin{abstract}
This paper proposes a traffic-flow evolutionary model under a dual updating mechanism that describes the day-to-day (DTD) dynamics of traffic flow and travel cost. To illustrate the concept, a simple two-route network is considered. Based on the nonlinear dynamic theory, the equilibrium stability condition of the system is derived and the condition for the division between the bifurcation and chaotic states of the system is determined. The characteristics of the DTD dynamic evolution of network traffic flow are investigated using numerical experiments. The results show that the system is absolutely stable when the sensitivity of travelers toward the route cost parameter $(\theta)$ is equal to or less than 0.923 . The bifurcation appears in the system when $\theta$ is larger than 0.923 . For values of $\theta$ equal to or larger than 4.402 , the chaos appears in the evolution of the system. The results also show that with the appearance of chaos, the boundary and interior crises begin to appear in the system when $\theta$ is larger than 6.773 and 10.403 , respectively. The evolution of network traffic flow is always stable when the proportion of travelers who do not change the route is $84 \%$ or greater.
\end{abstract}

Keywords: network traffic flow; dynamic evolution; stability; chaos

\section{Introduction}

With the rapid development of the social economy and the acceleration of urbanization, traffic congestion becomes widespread during peak hours in urban areas. The fundamental reason for this phenomenon is the imbalance of supply and demand, where the existing urban transportation infrastructure cannot meet the travel demand. Traffic congestion increases vehicle emissions, which contribute to air pollution. Two solutions to reduce traffic congestion are possible, namely: (1) building new transportation infrastructure and/or (2) optimizing the existing transportation system by means of traffic management and control. To achieve the desired results for either solution, it is necessary to master the day-to-day evolution characteristics of traffic flow on the transport network. Based on this cognition, traffic congestion may be alleviated, and in turn, the adverse environment may be reduced.

As it is known, traffic assignment focuses on how travelers choose routes and allocate the total origin-destination (OD) demand to each route, in order to obtain link flows that reflect the congestion degree of the road network. The results of traffic assignment are the basis for traffic planning and management decisions. Existing models, such as the user equilibrium (UE) model and the stochastic user equilibrium (SUE) model, concentrate on the final results of the equilibrium assignment of network traffic flows. However, the equilibrium model cannot explain how to achieve the equilibrium state. Actually, the travelers' day-to-day (DTD) route choice behavior is a dynamic non-cooperative 
game process that is long-term and repetitive. From a macroscopic viewpoint, this behavior leads to the DTD dynamic evolution process of network traffic flows [1-3].

Researchers have studied the DTD dynamic evolutionary characteristics of network traffic flows from two perspectives, namely: an individual perspective and an aggregate perspective. The individual perspective focuses on the choice behavior of individual travelers. After each traveler chooses a route, the route flows are aggregated in the network. On the other hand, considering all travelers as a system and handling them together, the aggregate perspective can be further classified as continuous or discrete. The continuous approach assumes that the number of days of network traffic flow is a continuous variable [4-7], while the discrete approach considers the number of days of network traffic flows as a discrete variable and is closer to reality. Typical references related to each perspective are presented in Table 1. For each reference, the table shows the type of analytical model considered, the updating variables (e.g., travel time, travel cost, and traffic flow), and the evolutionary elements analyzed (e.g., travelers' habituation, stability, bifurcation, and chaos), as well as any main findings. It is useful to present a brief review of the references in each category and to highlight the main differences among them.

In the individual perspective, a microscopic numerical simulation or a laboratory experiment is adopted. For example, Nakayama et al. [8] developed a drivers' route choice model based on the hypothesis that drivers were not rational or homogeneous, and concluded that the final convergence state of the network did not necessarily converge to UE through microscopic simulation. Klügl et al. [9] designed an adaptive updating rule to describe the DTD route choice behavior based on an agent simulation environment. The results showed that the traffic flow distribution could converge to UE. Selten et al. [10] conducted laboratory experiments of a DTD route choice game with two parallel roads by selecting 18 college students as subjects, and reported that large fluctuations do not diminish with individual experiences. Kim et al. [11] employed a DTD evolutionary approach and developed agent-based simulation models that included a drivers' learning model, preference model, and preference sensitivity. The results showed that the assumption of perfect information is the most influencing on traffic assignment results. Recently, Zhang et al. [12] developed a DTD route choice model with social interaction information from friends, and then conducted a laboratory experiment. The experimental results show that a larger proportion of social interactions do not necessarily lead to better route choice results either for individuals or for the whole system.

Research related to the aggregate-continuous perspective has two limitations, (1) the continuoustime approach is not plausible in reality and (2) homogeneous population assumptions require additional dispersion modules. Extensive research related to the aggregate-discrete perspective has been conducted. Typical publications include Cantarella and Cascetta [13], who developed DTD dynamic models for both deterministic and stochastic process. They proposed conditions for the existence, uniqueness, and stability of equilibrium in the deterministic model, and proposed conditions for stochastic process regularity in the stochastic model. Zhang and Jarrett [14] developed a dynamic model based on the conventional gravity model, which described the variations of OD flows over discrete-time periods. The authors showed that chaos occurs when the system dimension is relatively high. Watling and Hazelton [15] proposed a DTD dynamic evolutionary model considering the route flow and travel cost as basic variables. The authors derived the stability condition for the network equilibrium using a simple example. Guo and Huang [16] developed a dynamic evolutionary model of traffic assignment with endogenous OD demands. The model stability was analyzed and the resultant UE was shown to be stable under certain conditions. Bie and Lo [17] investigated the stability of UE pursued by a DTD adjustment process, and provided tools to determine the stability of the equilibrium and to estimate its domain of attraction. Subsequently, further research has been conducted by He et al. [18,19], Han and Du [20], Guo et al. [21], Rambha and Boyles [22], Ye et al. [23], and Zhou et al. [24]. Recently, Cantarella and Watling [25] presented a unified approach for both discrete and continuous-time models. 
Table 1. Characteristics of existing and proposed day-to-day dynamic traffic evolution models.

\begin{tabular}{|c|c|c|c|}
\hline Evolution Type & Reference & $\begin{array}{l}\text { Updating } \\
\text { Variables }\end{array}$ & Analyzed Elements/Findings \\
\hline \multirow{5}{*}{ Individual } & Nakayama et al. [8] & $\mathrm{TT}^{\mathrm{a}}$ & $\mathrm{UE}^{\mathrm{a}}$ is not necessarily reached \\
\hline & Klügl et al. [9] & Reward & UE can be reached \\
\hline & Selten et al. [10] & Payoff & UE cannot be reached \\
\hline & Kim et al. [11] & TT & $\begin{array}{l}\text { Perfect information has the most effect on route } \\
\text { assignment }\end{array}$ \\
\hline & Zhang et al. [12] & TT & $\begin{array}{l}\text { Social information is not necessarily beneficial for } \\
\text { individuals or the whole system }\end{array}$ \\
\hline \multirow{4}{*}{$\begin{array}{l}\text { Aggregate } \\
\text { (Continuous) }\end{array}$} & Smith and Watling [4] & $\mathrm{TF}^{\mathrm{a}}$ & UE can be reached \\
\hline & Guo et al. [5] & $\mathrm{TT}, \mathrm{TF}$ & $\begin{array}{c}\text { Proved equivalent, uniqueness, and stability of } \\
\text { stationary points }\end{array}$ \\
\hline & Cho and Hwang [6] & $\mathrm{TT}, \mathrm{TF}$ & Proved uniqueness of stationary state \\
\hline & Mounce [7] & $\mathrm{TT}, \mathrm{TF}$ & Derived global convergence condition for UE \\
\hline \multirow{15}{*}{$\begin{array}{l}\text { Aggregate } \\
\text { (Discrete) }\end{array}$} & Cantarella and Cascetta [13] & $\mathrm{TT}, \mathrm{TF}$ & $\begin{array}{l}\text { Proposed conditions for stability of } \\
\text { stationary points }\end{array}$ \\
\hline & Zhang and Jarrett [14] & $\mathrm{TC}^{\mathrm{a}}$ & Chaos occurs when system dimension is high \\
\hline & Watling et al. [15] & TC & Derived condition for stability of SUE ${ }^{a}$ \\
\hline & Guo and Huang [16] & TC & Derived condition for stability of UE \\
\hline & Bie and Lo [17] & TC & Analyzed stability and attraction domain of UE \\
\hline & He and Liu $[18,19]$ & TC & Proposed correction model for equilibrium \\
\hline & Han and Du [20] & $\mathrm{TC}, \mathrm{TF}$ & Proposed and analyzed a link-based model \\
\hline & Guo and Yang [21] & TC, TF & Proved unique. and convergence of stationary state \\
\hline & Rambha and Boyles [22] & TC, TF & Dynamic pricing reduced total system travel time \\
\hline & Ye et al. [23] & $\mathrm{TC}$ & $\begin{array}{l}\text { Analyzed effect of path flow/time on } \\
\text { route switching }\end{array}$ \\
\hline & Zhou et al. [24] & $\mathrm{TF}$ & $\begin{array}{l}\text { Analyzed the convergence of day-to-day flow } \\
\text { dynamic model to mixed equilibrium state }\end{array}$ \\
\hline & Liu et al. [1,2], Li et al. [3] & $\mathrm{TC}$ & Analyzed bifurcation, stability, and chaos \\
\hline & Cantarella [26] & TC, TF & Analyzed bifurcation and stability \\
\hline & Zhao and Orosz [27] & $\mathrm{TC}, \mathrm{TF}$ & $\begin{array}{l}\text { Analyzed travelers' habituation, stability, } \\
\text { and bifurcation }\end{array}$ \\
\hline & $\begin{array}{l}\text { Proposed model in this } \\
\text { paper }\end{array}$ & $\mathrm{TC}, \mathrm{TF}$ & $\begin{array}{l}\text { Analyzed travelers' habituation, stability, } \\
\text { bifurcation, and chaos. Derived stability condition }\end{array}$ \\
\hline
\end{tabular}

a $\mathrm{TT}$ = travel time; TC = travel cost; TF = traffic flow; UE = user equilibrium; SUE = stochastic user equilibrium.

Liu et al. [1,2] investigated the DTD dynamic evolution of the network traffic flow with fixed and elastic demand. Li et al. [3] developed a bounded rational binary logit (BRBL) model to describe travelers' route-choice behavior. The preceding authors derived the stability condition of network traffic flow evolution and focused on the bifurcation and chaos phenomena when the system was unstable. However, they considered only the DTD updating of travel cost and did not consider the travelers' habituation. On the other hand, the DTD dynamic assignment model by Cantarella [26], and Zhao and Orosz [27] considered updating both the travel cost and traffic flow, but analyzed only the stability and bifurcation behavior (the chaos phenomena was not analyzed). Clearly, there is a need to develop a model of the entire evolution spectrum (stability-bifurcation-chaos) under a dual updating mechanism, considering traveler's habits.

What are the characteristics of the evolution of network traffic flow when travelers' habits are considered? And what are the characteristics of chaos when the evolution is unstable? To answer these questions, taking the two-route network as the research object, this paper formulates a traffic flow evolutionary model under a dual updating mechanism considering travelers' habits. The conditions 
for the equilibrium stability of the system and the division between the bifurcation and chaotic states of the system are established. The chaotic phenomenon in the DTD dynamic evolution of the network traffic flow is emphatically analyzed. The rest of this paper is organized as follows. In Section 2, we present the traffic flow evolutionary model under the dual updating mechanism of flow and travel cost. The final state of the network traffic flow evolution and critical condition are presented in Section 3. Numerical experiments and related insights are presented in Section 4. Some conclusions are drawn in Section 5.

\section{Mathematical Model}

Consider a simple network that consists of one OD pair connected by two parallel routes. The total demand between the single OD pair is $d$. Let $f_{1}^{(n)}$ and $f_{2}^{(n)}$ denote the actual flows and $\bar{C}_{1}^{(n)}$ and $\bar{C}_{2}^{(n)}$ denote the actual travel costs of Routes 1 and 2 on day $n$, respectively. It is assumed that the travel cost of each route is related only to the traffic flow of the route. The travel cost functions are expressed as $\bar{C}_{1}^{(n)}=g_{1}\left(f_{1}^{(n)}\right)$ and $\bar{C}_{2}^{(n)}=g_{2}\left(f_{2}^{(n)}\right)$.

According to assumptions of the SUE principle, let $\hat{C}_{k}^{(n)}=C_{k}^{(n)}+\varepsilon_{k}$ be the perceived travel cost of route $k(k=1,2)$ on day $n$, where $C_{k}^{(n)}$ and $\varepsilon_{k}$ are the deterministic and random components, respectively. It is assumed that $\varepsilon_{1}$ and $\varepsilon_{2}$ are independent of each other and identically distributed Gumbel. The deterministic component of the perceived travel cost is used subsequently. Then, according to the authors of [28], the route-choice probabilities for Routes 1 and 2 on day $n$ are expressed as follows:

$$
\begin{gathered}
P_{1}{ }^{(n)}=\frac{1}{1+e^{\theta\left(C_{1}^{(n)}-C_{2}^{(n)}\right)}}, \\
P_{2}^{(n)}=1-P_{1}^{(n)}=\frac{1}{1+e^{-\theta\left(C_{1}^{(n)}-C_{2}^{(n)}\right)}},
\end{gathered}
$$

where $\theta$ is a parameter associated with the traveler's characteristic, and describes the random degree of their route choice $(\theta>0)$. The greater $\theta$ is, the lower the randomness of the travelers' route choice is, and the more sensitive travelers are to the route cost, and vice versa. According to the SUE principle, the traffic flow on day $n$ is assigned according to the logit model as follows:

$$
\begin{gathered}
{\overline{f_{1}}}^{(n)}=d \cdot P_{1}^{(n)}=\frac{d}{1+e^{\theta\left(C_{1}^{(n)}-C_{2}^{(n)}\right)},} \\
{\overline{f_{2}}}^{(n)}=d-{\overline{f_{1}}}^{(n)},
\end{gathered}
$$

where ${\overline{f_{1}}}^{(n)}$ and ${\overline{f_{2}}}^{(n)}$ represent the traffic flows assigned by the logit model for Routes 1 and 2 on day $n$, respectively.

In reality, there are a certain number of travelers who do not change the route because of their preferences and habits in daily travel. Let the percentage of travelers who do not change the route be denoted by $\rho$. Then, the actual traffic flow on day $n$ is updated according to the actual traffic flows on day $(n-1)$, and those assigned by the logit model on day $n$, which is expressed as follows:

$$
\begin{gathered}
f_{1}^{(n)}=\rho f_{1}^{(n-1)}+(1-\rho){\overline{f_{1}}}^{(n)}, \\
f_{2}^{(n)}=d-f_{1}^{(n)},
\end{gathered}
$$

where $\rho$ is the habituation weight coefficient, and $\rho \in[0,1)$. The parameter $\rho$ reflects the habituation of route-choice behavior. In other words, a part of travelers still choose the route of the previous day, following their habituation. A larger $\rho$ means that more travelers do not change the route, 
and vice versa. Equation (5) describes the evolution of traffic flow, which has not been considered in Liu et al. [1,2] or Li et al. [3].

The perceived travel cost on day $n$ is updated using the perceived and actual travel cost on day $(n-1)$, which is expressed as follows:

$$
\begin{gathered}
C_{i}^{(n)}=\varphi C_{i}^{(n-1)}+(1-\varphi){\overline{C_{i}}}^{(n-1)}(i=1,2), \\
C_{i}^{(n)}=\varphi C_{i}^{(n-1)}+(1-\varphi) g_{i}\left(f_{i}^{(n-1)}\right)(i=1,2),
\end{gathered}
$$

where $\varphi$ is the cost weight coefficient and $\varphi \in[0,1)$. The parameter $\varphi$ reflects the traveler's dependence on the actual travel cost of the previous day. A larger $\varphi$ means that travelers are less dependent on the actual travel cost of the previous day, and vice versa.

Thus, the DTD dynamic evolutionary model of network traffic flows is obtained as follows:

$$
\begin{gathered}
C_{1}^{(n)}=\varphi C_{1}^{(n-1)}+(1-\varphi) g_{1}\left(f_{1}^{(n-1)}\right), \\
C_{2}^{(n)}=\varphi C_{2}^{(n-1)}+(1-\varphi) g_{2}\left(d-f_{1}^{(n-1)}\right), \\
f_{1}^{(n)}=\rho f_{1}^{(n-1)}+(1-\rho){\overline{f_{1}}}^{(n)},
\end{gathered}
$$

Let $\widetilde{C}^{(n)}=C_{1}^{(n)}-C_{2}^{(n)}$. From Equations (1) and (2), in view of the fact that route-choice probability depends only on the difference in route cost, the preceding model may be further simplified to a two-dimensional system by subtracting Equation (9) from Equation (10), as follows:

$$
\widetilde{C}^{(n)}=\varphi \widetilde{C}^{(n-1)}+(1-\varphi) g_{1}\left(f_{1}^{(n-1)}\right)-(1-\varphi) g_{2}\left(d-f_{1}^{(n-1)}\right) .
$$

\section{Evolution State and Critical Condition}

Cantarella and Cascetta [13] proposed the stability conditions of general networks, but the derivation process is complicated. Relatively simple stability conditions and proof methods are provided as follows.

Assumption: The functions of travel cost about path flow are monotonically increasing and continuously differentiable.

This assumption reflects the characteristic of the road impedance function and conforms to the actual traffic flow characteristics. This assumption is also usually used in the study of network traffic flows. Before a theorem for the stability condition and its proof, it is useful to first present Lemma 1, whose proof is presented elsewhere [29].

Lemma 1. The sufficient and necessary condition that the modulus of the two roots of the quadratic equation $r^{2}+p_{1} r+p_{2}>0$ are both less than 1 is [29]

$$
1+p_{1}+p_{2}>0,1-p_{1}+p_{2}>0,1-p_{2}>0
$$

At the equilibrium point, let the evolution Equations (11) and (12) be written as $\widetilde{C}^{(n)}=\widetilde{C}^{*}$, $f_{1}^{(n)}=f_{1}^{*}, P_{1}^{\prime}=-\left.\frac{\partial P_{1}^{(n)}}{\partial \widetilde{C}^{(n)}}\right|_{\widetilde{C}^{*}}, g_{1}^{\prime}=\left.\frac{d g_{1}\left(f_{1}\right)}{d f_{1}}\right|_{f_{1}^{*}} g_{2}^{\prime}=-\left.\frac{d g_{2}\left(d-f_{1}\right)}{d f_{1}}\right|_{f_{1}^{*}}$.

Theorem 1. At the equilibrium point, the condition that the DTD dynamic assignment system is asymptotically stable in a two-parallel route network is

$$
d P_{1}^{\prime}\left(g_{1}^{\prime}+g_{2}^{\prime}\right)<\frac{(1+\varphi)(1+\rho)}{(1-\varphi)(1-\rho)}
$$


Proof. The Jacobian matrix of dynamical system of Equations (11) and (12) is

$$
\boldsymbol{J}=\left(\begin{array}{ll}
\frac{\partial \widetilde{C}^{(n)}}{\partial \widetilde{C}^{(n-1)}} & \frac{\partial \widetilde{C}^{(n)}}{\partial f_{1}^{(n-1)}} \\
\frac{\partial f_{1}^{(n)}}{\partial \widetilde{C}^{(n-1)}} & \frac{\partial f_{1}^{(n)}}{\partial f_{1}^{(n-1)}}
\end{array}\right) .
$$

At the equilibrium point, the characteristic equation of the matrix $J$ is $\lambda^{2}-$ $\left[\varphi+\rho-(1-\varphi)(1-\rho) d P_{1}^{\prime}\left(g_{1}^{\prime}+g_{2}^{\prime}\right)\right] \lambda+\varphi \rho=0 . \quad$ The roots of the characteristic equation are $\lambda_{1}, \lambda_{2}$. According to the stability theory of nonlinear dynamics, it can be known that the modulus of two roots of the characteristic equation of the matrix $J$ are both less than 1 . Then, by considering Lemma 1, the stability conditions can be formulated as

$$
\begin{gathered}
1-\left[\varphi+\rho-(1-\varphi)(1-\rho) d P_{1}^{\prime}\left(g_{1}^{\prime}+g_{2}^{\prime}\right)\right]+\varphi \rho>0 \\
1+\varphi+\rho-(1-\varphi)(1-\rho) d P_{1}^{\prime}\left(g_{1}^{\prime}+g_{2}^{\prime}\right)+\varphi \rho>0 \\
1-\varphi \rho>0
\end{gathered}
$$

where $P_{1}^{\prime}>0, g_{1}^{\prime}>0, g_{2}^{\prime}>0, \varphi \in[0,1)$, and $(1-\varphi)(1-\rho)\left[1+d P_{1}^{\prime}\left(g_{1}^{\prime}+g_{2}^{\prime}\right)\right]>0$. It can be obviously shown that Equation (17) is always satisfied. Equation (15) can be easily transformed into $(1-\varphi)(1-\rho)\left[1+d P_{1}^{\prime}\left(g_{1}^{\prime}+g_{2}^{\prime}\right)\right]>0$. Thus, Equation (15) is also satisfied consistently. Equation (16) can be transformed into $d P_{1}^{\prime}\left(g_{1}^{\prime}+g_{2}^{\prime}\right)<\frac{(1+\varphi)(1+\rho)}{(1-\varphi)(1-\rho)}$. Thus, Theorem 1 has been proved.

As $\varphi \in[0,1)$ and $\rho \in[0,1)$, it is not difficult to derive the following Corollary 1 from Theorem 1 .

Corollary 1. If $d P_{1}^{\prime}\left(g_{1}^{\prime}+g_{2}^{\prime}\right)<1$, at the equilibrium point, the DTD dynamic evolutionary system of traffic flow is asymptotically stable in a two-route parallel network, regardless of the values that the parameters $\rho$ and $\varphi$ take within the interval $[0,1)$.

Corollary 1 shows that as long as the traffic system satisfies certain conditions, the DTD dynamic evolutionary system of network traffic flows is asymptotically stable at the equilibrium point, no matter how much information the travelers depend on and how many people do not change the route.

It can be seen from Theorem 1 that bifurcation or chaos may appear in the traffic flow evolution of the road network when Equation (13) is not satisfied. In order to determine whether the system is in a chaotic state, we need to solve the Lyapunov exponent (LE) of the system [30]. If LE is greater than 0, the system is in a chaotic state. Then, the LE of the dynamic system of Equations (11) and (12) is

$$
L_{i}=\lim _{n \rightarrow \infty} \frac{1}{n} \log \left|\lambda_{i}\right|, i=1,2 .
$$

where $\lambda_{1}$ and $\lambda_{2}$ are the roots of the characteristic equation of the matrix $J$.

The maximum LE of the dynamic system is

$$
L=\max \left(L_{1}, L_{2}\right)
$$

The condition for the division of the dynamic system into three states, stability, bifurcation (periodic motion), and chaos, are summarized in Table 2. 
Table 2. Day-to-day dynamical evolution state's condition for division.

\begin{tabular}{cc}
\hline Final Evolution State & Condition \\
\hline Stability and convergence to equilibrium point & $d P_{1}^{\prime}\left(g_{1}^{\prime}+g_{2}^{\prime}\right)<\frac{(1+\varphi)(1+\rho)}{(1-\varphi)(1-\rho)}$ \\
Bifurcation (periodic motion) & $d P_{1}^{\prime}\left(g_{1}^{\prime}+g_{2}^{\prime}\right) \geq \frac{(1+\varphi)(1+\rho)}{(1-\varphi)(1-\rho)}$ and $L \leq 0$ \\
Chaotic motion & $L>0$ \\
\hline
\end{tabular}

From the preceding analysis, we can see that the dynamic system will be stable under certain conditions (Theorem 1), otherwise bifurcation or chaotic motion will occur. Subsequently, numerical experiments are conducted to investigate the effect of the parameters on the DTD dynamic evolution of network traffic flows.

\section{Numerical Experiments}

\subsection{Network Description}

We consider the same traffic network, which includes two routes. The travel time is calculated for each route as a function of traffic flow using the following Bureau of Public Roads (BPR) function,

$$
\left.t=t_{f}\left[1+0.15(q / C)^{4}\right)\right]
$$

where $t$ is the actual travel time, $t_{f}$ is free-flow link travel time, $q$ is link traffic flow, and $C$ is the link capacity. It is noted that each route contains one link. The following data are considered: $t_{f 1}=22 \mathrm{~min}$, $C_{1}=1500 \mathrm{pcu} / \mathrm{h}$, and $t_{f 2}=25 \mathrm{~min}, C_{2}=2000 \mathrm{pcu} / \mathrm{h}$, and $d=1500 \mathrm{pcu} / \mathrm{h}$. We assume that the travel cost is measured only by travel time. In the network, when the OD demand is fully loaded onto Route 1 , the travel time of two routes is $25.3 \mathrm{~min}$ and $25 \mathrm{~min}$, respectively. When the OD demand is fully loaded onto Route 2, the travel time of two routes is $22 \mathrm{~min}$ and $26.2 \mathrm{~min}$, respectively. Substituting the initial value into Equations (11) and (12), then the evolutionary model can be obtained as

$$
\begin{aligned}
\widetilde{C}^{(n)} & =\varphi \widetilde{C}^{(n-1)}+(1-\varphi) t_{f 1}\left[1+0.15\left(\frac{f_{1}^{(n-1)}}{C_{1}}\right)^{4}\right], \\
& -(1-\varphi) t_{f 2}\left[1+0.15\left(\frac{d-f_{1}^{(n-1)}}{C_{2}}\right)^{4}\right] \\
& f_{1}^{(n)}=\rho f_{1}^{(n-1)}+(1-\rho) \cdot \frac{d}{1+e^{\theta \widetilde{C}^{(n)}}} .
\end{aligned}
$$

\subsection{Evolution Characteristics}

The dynamic evolution characteristics of the system of Equations (21) and (22) are discussed in this section, when $\rho, \theta$, and $\varphi$ take different values, where $\theta \in(0,+\infty), \rho \in[0,1)$, and $\varphi \in[0,1)$. According to Corollary 1 , the stability of the equilibrium point is in a critical situation when $d P_{1}^{\prime}\left(g_{1}^{\prime}+g_{2}^{\prime}\right)=1$. At this point, $\theta=0.923$ can be calculated, which is the same as that by Liu et al. [1].

Case 1: $\theta \leq 0.923$

For $\theta \leq 0.923$, the equilibrium point is asymptotically stable, regardless of the values that $\rho$ and $\varphi$ take. For example, for $\theta=0.8, \rho=0.5$ (Figure 1a), the relation between the flow bifurcation and $\varphi$ will be constant, where the flow of Route 1 is $1192 \mathrm{pcu} / \mathrm{h}$. Similarly, for $\theta=0.8$ and $\varphi=0.5$ (Figure 1b), the relation between the flow bifurcation and $\rho$ will be constant, where the flow of Route 1 is also $1192 \mathrm{pcu} / \mathrm{h}$. 


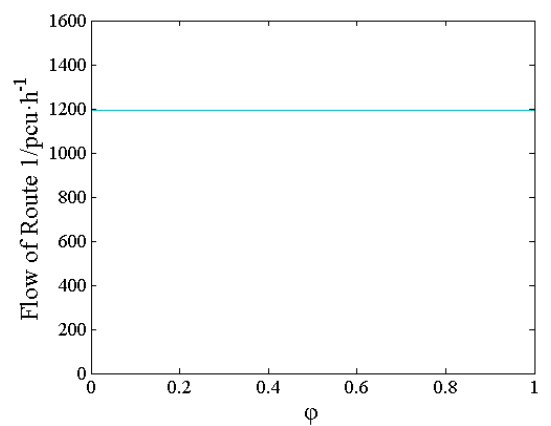

(a) $\rho=0.5$

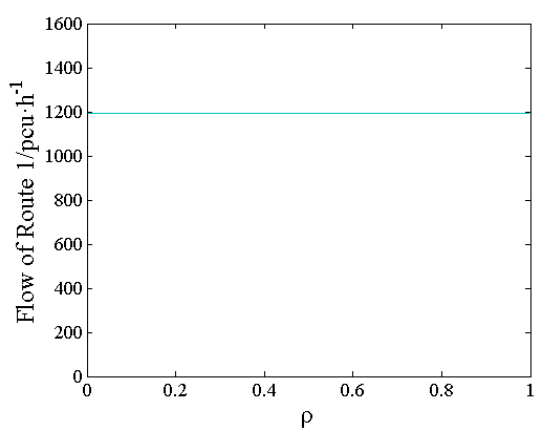

(b) $\varphi=0.5$

Figure 1. Variation of flow bifurcation diagram when $\theta=0.8$.

Case 2: $0.923<\theta \leq 4.402$

We fix $\theta=4$ and discuss the bifurcation variation of the flow of Route 1 when $\rho$ and $\varphi$ take different values (Figure 2). Consider first the variation of the flow bifurcation with $\varphi$, when $\rho$ takes different values. For $\rho=0$ (all travelers change routes), the bifurcation sequence of the system is "stability to period-2 orbit", as $\varphi$ decreases (Figure 2a). Then, the bifurcation sequence is "stability to period-2 orbit to period-4 orbit" as $\rho$ increases (Figure 2b). From the horizontal view, the system is stable in a large area and the bifurcation point evolves to the left. As $\rho$ increases to 0.4 , the bifurcation sequence is "stability to period-2 orbit" again, and the bifurcation point evolves further to the left (Figure 2c). The system is always stable when $\rho$ is greater than 0.497 , regardless of the value of $\varphi \in[0,1)$.

Now consider the variation of flow bifurcation with $\rho$ when $\varphi$ takes different values. For $\varphi=0$ (all travelers rely on the actual cost for travel), the bifurcation sequence of the system is "stability to period-doubling bifurcation to period-halving bifurcation" as $\rho$ decreases (Figure 2e). Finally, period-2 orbit occurs at $\rho=0$. Then, the bifurcation sequence is only "stability to period-2 orbit" as $\varphi$ increases (Figure 2f,g). Moreover, the stability region becomes larger and larger, and the bifurcation point gradually moves to the left. However, the system is always stable when $\varphi$ is greater than 0.497 , regardless of the value of $\rho \in[0,1)$. It can be noted that the system is always stable when parameter $\rho$ or $\varphi$ is greater than 0.497 . This indicates that these two parameters have the same effect on the stability of the system.

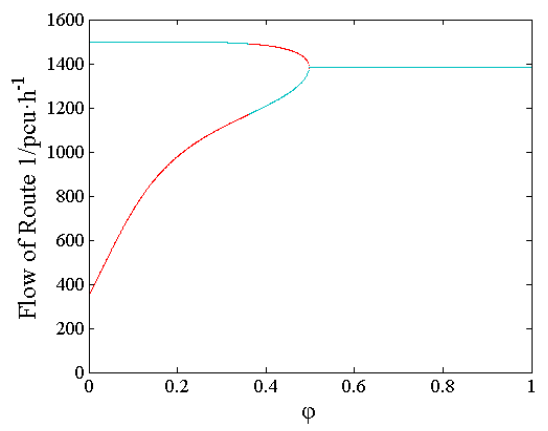

(a) $\rho=0$

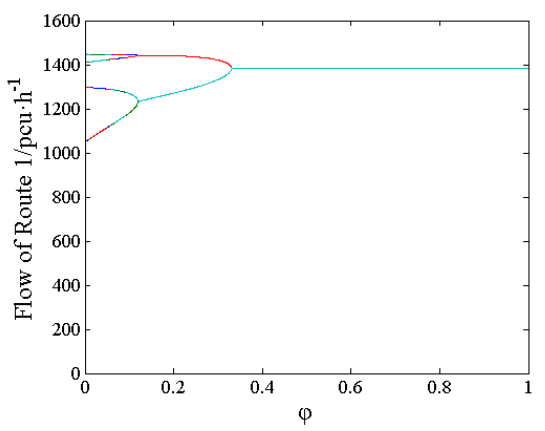

(b) $\rho=0.2$

Figure 2. Cont. 


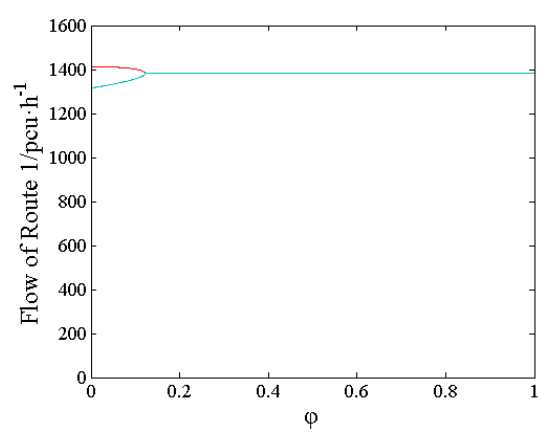

(c) $\rho=0.4$

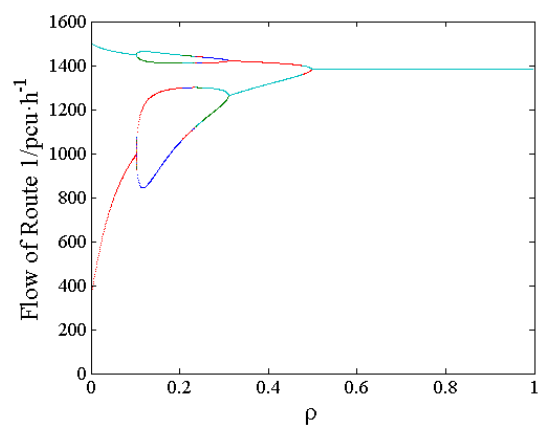

(e) $\varphi=0$

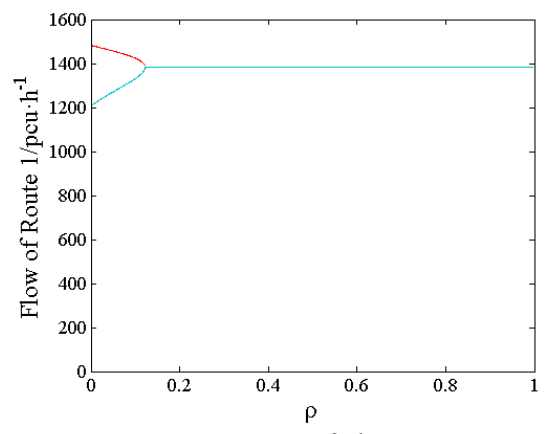

(g) $\varphi=0.4$

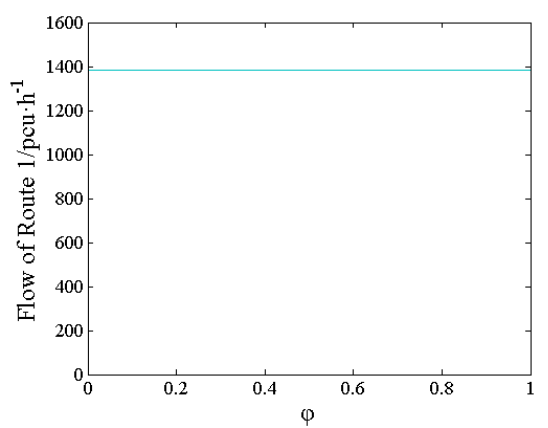

(d) $\rho=0.5$

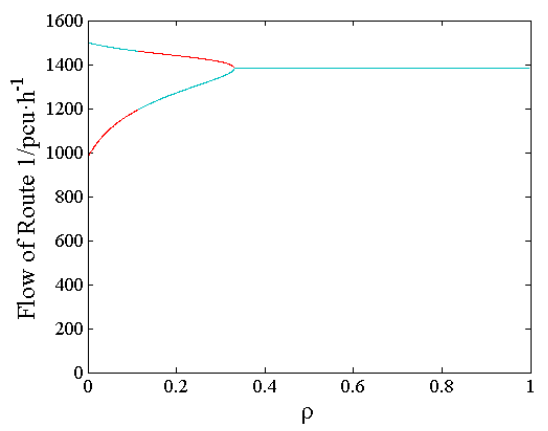

(f) $\varphi=0.2$

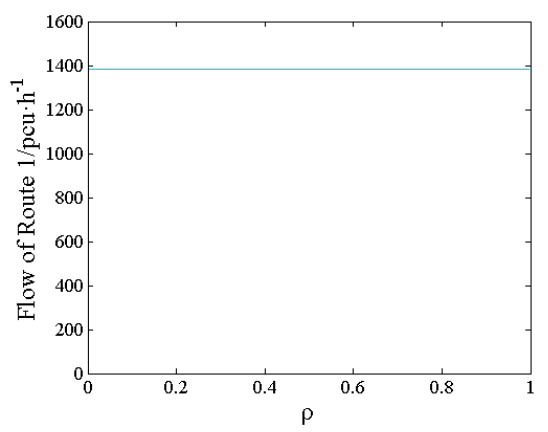

(h) $\varphi=0.5$

Figure 2. Variation of flow bifurcation diagram when $\theta=4$.

Case 3: $\theta>4.402$

Many numerical experiments have shown that the Lyapunov exponent of the system will be larger than 0 when $\theta>4.402$, where the chaotic phenomena will appear in the dynamic system. Taking $\theta=5$ as an example, the influence of $\rho$ and $\varphi$ on the chaos is discussed (Figure 3). For $\rho=0.2$, the variations of the bifurcation of flow of Route 1 and the LE with $\varphi$ are shown in Figure 3a,b, respectively. As noted, the bifurcation sequence of the system is "stability to period-doubling bifurcation to chaos" as $\varphi$ decreases. Finally, the chaotic motion occurs at $\varphi=0$. For $\varphi=0$, the variation of the bifurcation of flow of Route 1 and the LE with $\rho$ are shown in Figure $3 c, d$, respectively. As noted, the bifurcation sequence is "stability to period-doubling bifurcation to chaos to period-halving bifurcation" as $\rho$ decreases. Eventually, the period-doubling motion occurs at $\rho=0$, coinciding with the conclusions of Liu et al. [1]. 


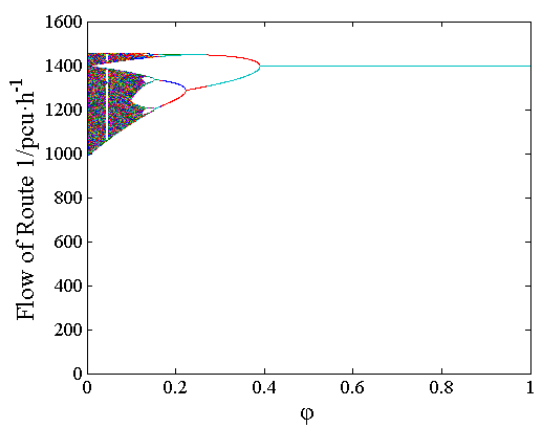

(a) $\rho=0.2$ bifurcation diagram

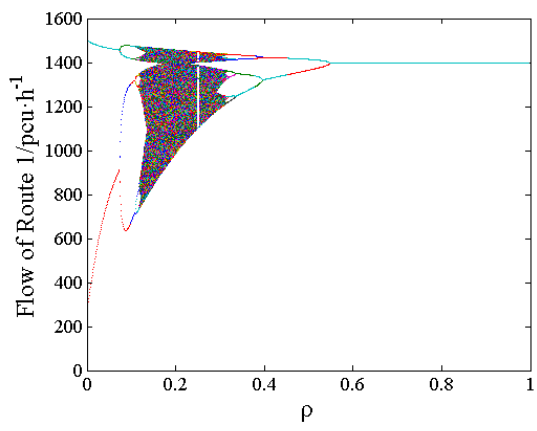

(c) $\varphi=0$ bifurcation diagram

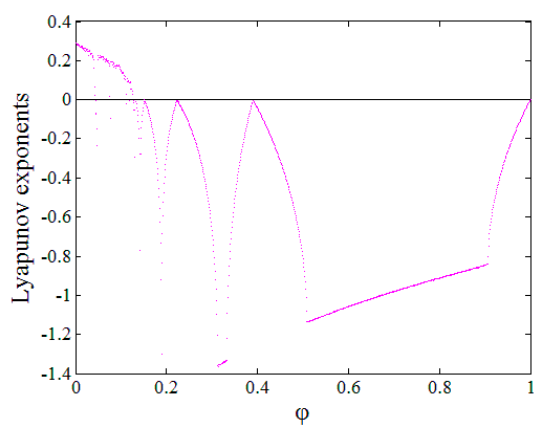

(b) $\rho=0.2$ Lyapunov exponent

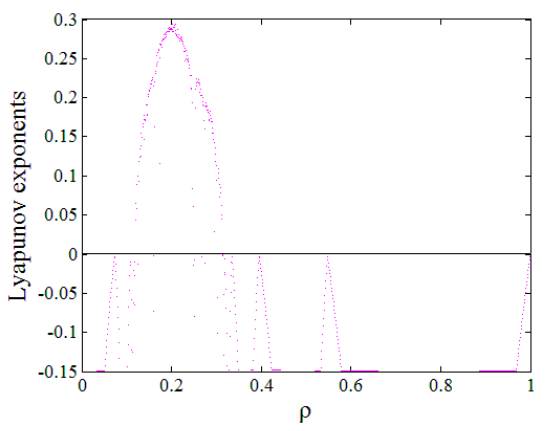

(d) $\varphi=0$ Lyapunov exponent

Figure 3. Variation of flow bifurcation diagram and the Lyapunov exponent of the system when $\theta=5$.

The evolution state can be also verified through the time series and power spectrum, which can be obtained by fast Fourier transform (FFT). When $\theta=5$, for $\rho=0.2, \varphi=0.2$, or 0 , the flow of Route 1 with time and its power spectrum are shown in Figure 4. For $\rho=0.2$ and $\varphi=0.2$, it can be seen from Figure $4 \mathrm{a}$ that the evolution is periodic. Seen from Figure $4 \mathrm{~b}$, only the power spectrum of frequency $1 / 4$ is large, and the others are small. Therefore, we can deduce that the evolution is periodic with period 4. For $\rho=0.2$ and $\varphi=0$, Figure 4c shows that the flow evolution is irregular. The spectrum is broadband and has a broad peak from Figure $4 \mathrm{~d}$, so we can deduce that the evolution is chaotic. However, in practice, it is difficult to distinguish the very noisy data from chaotic behavior using power spectrum. Therefore, the Lyapunov exponent is used next to distinguish whether the evolution is chaotic.

The boundary crisis appears in the dynamic evolution of the system when $\theta>6.773$ (the explanation of the boundary crisis can be referred to Lim and Kim [31], and Jiang et al. [32]). Taking $\theta=9$ as an example, for $\rho=0.3$, the variation of the bifurcation of flow of Route 1 with $\varphi$ is shown in Figure 5a. The boundary crisis obviously appears in the system at $\varphi=0.2$ and $\varphi=0.27$. For $\varphi=0.2$, the boundary crisis obviously appears in the system at $\rho=0.3, \rho=0.34$, and $\rho=0.38$ (Figure 5c). In Figure 5b,d, the crisis is confirmed by LE, which suddenly changes at two and three points, respectively. 


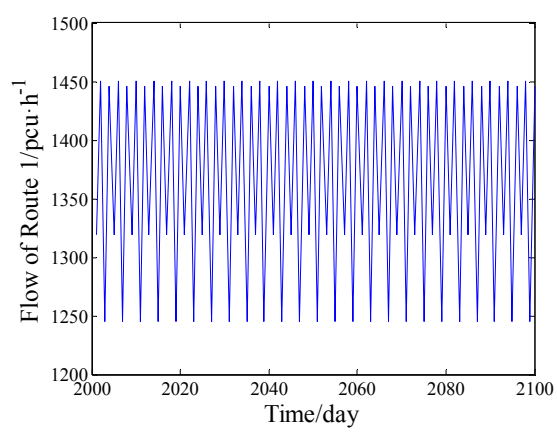

(a) $\rho=0.2, \varphi=0.2$ flow of route 1 with time

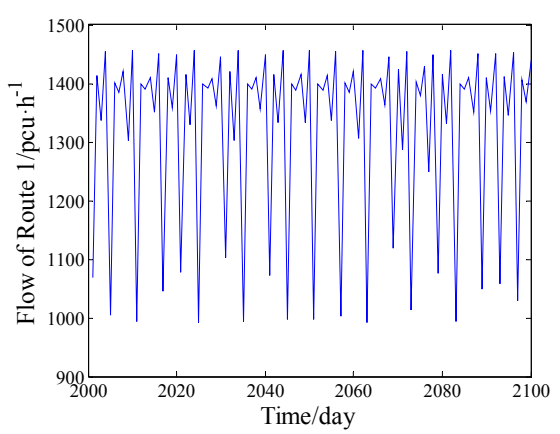

(c) $\rho=0.2, \varphi=0$ flow of route 1 with time

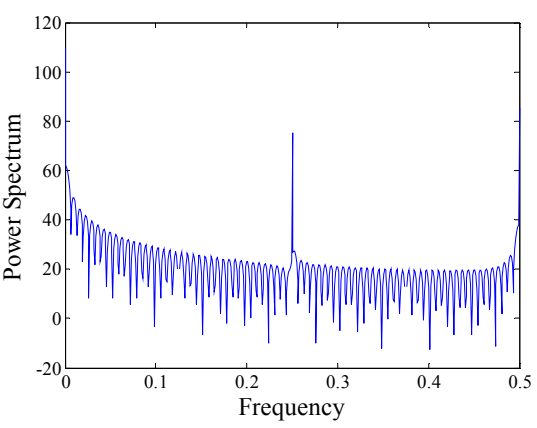

(b) power spectrum

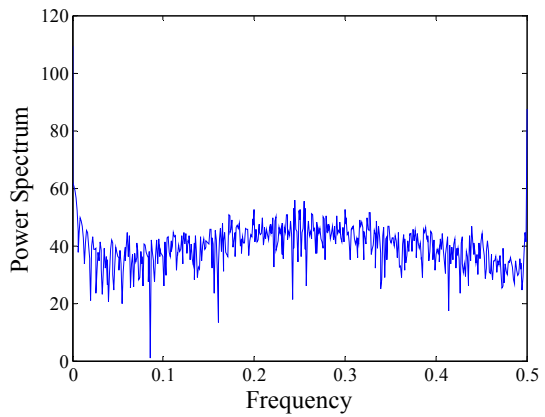

(d) power spectrum

Figure 4. Time series and power spectrum when $\theta=5$

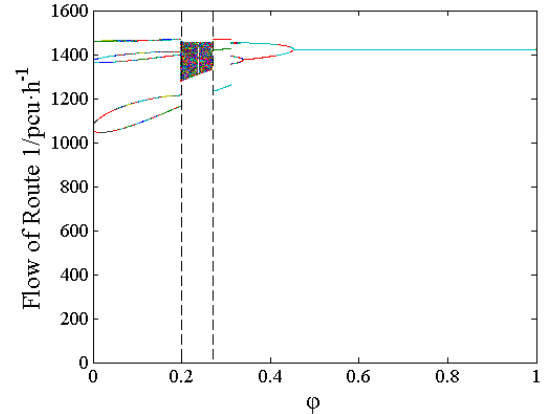

(a) $\rho=0.3$ bifurcation diagram

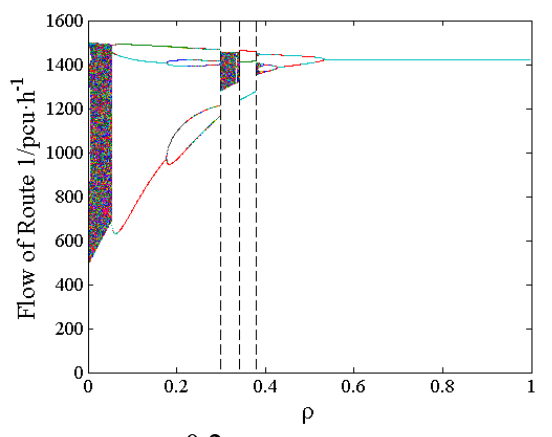

(c) $\varphi=0.2$ bifurcation diagram

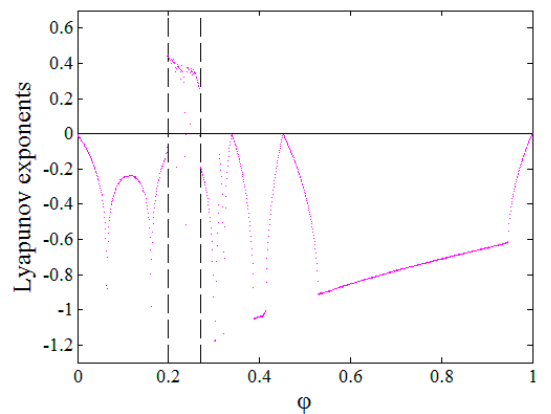

(b) $\rho=0.3$ Lyapunov exponent

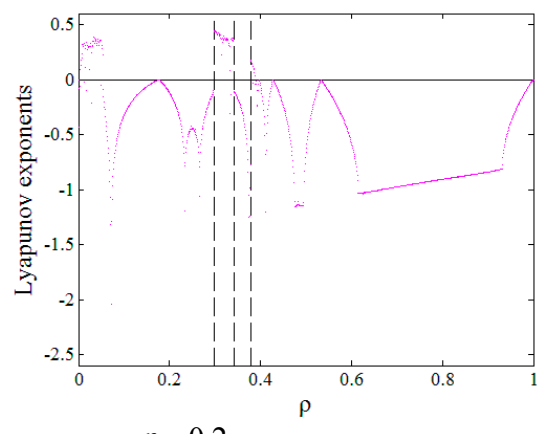

(d) $\varphi=0.2$ Lyapunov exponent

Figure 5. Variation of the flow bifurcation diagram and Lyapunov exponent of the system when $\theta=9$.

The interior crisis appears in the dynamic evolution of the system when $\theta>10.403$ (an explanation of the interior crisis can be referred to Lim and Kim [31] and Jiang et al. [32]). Taking $\theta=11$ as an example, for $\rho=0.4$, the variation of the bifurcation of flow of Route 1 with $\varphi$ is shown in Figure 6a. The interior crisis obviously appears in the system's chaotic region at $\varphi=0.06, \varphi=0.11, \varphi=0.18$, and $\varphi=0.19$. In Figure $6 \mathrm{~b}$ the crisis is also confirmed by LE, which suddenly changes at two points 
( $\varphi=0.18$ and $\varphi=0.19$ ). As shown in Figure $6 \mathrm{c}$, for $\varphi=0.3$, the boundary and interior crises appear in the system at the same time, where the interior and boundary crises appear in the system at $\rho=0.3$ and $\rho=0.36$, respectively. The crisis is also confirmed by LE (Figure $6 \mathrm{~d}$ ).

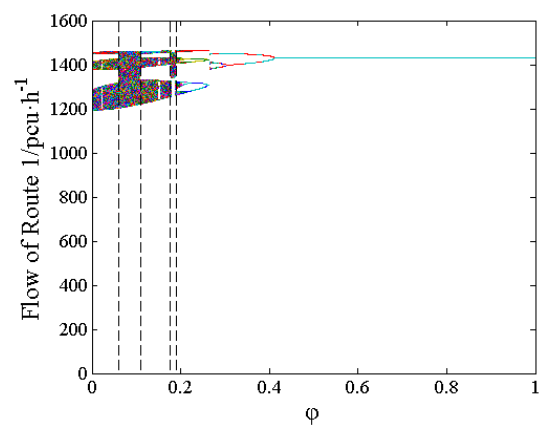

(a) $\rho=0.4$ bifurcation diagram

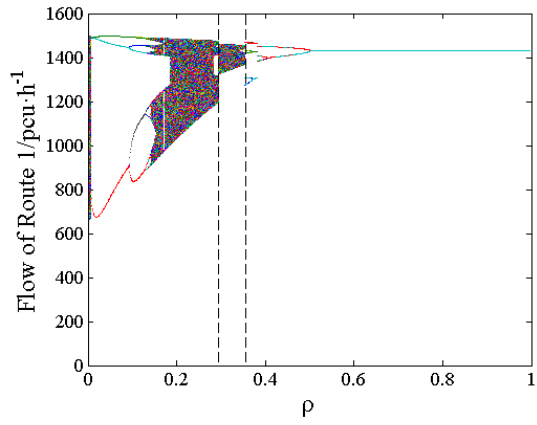

(c) $\varphi=0.3$ bifurcation diagram

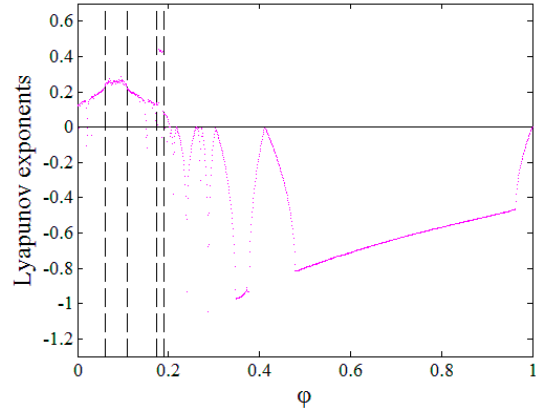

(b) $\rho=0.4$ Lyapunov exponent

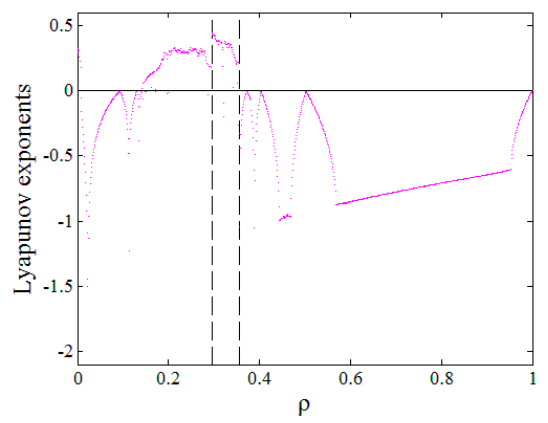

(d) $\varphi=0.3$ Lyapunov exponent

Figure 6. Variation of flow bifurcation diagram and Lyapunov exponent of the system when $\theta=11$.

The larger the parameter of $\theta$, the more obvious the chaotic motion is. Figure 7 shows the variation of the bifurcation of flow of Route 1 with $\varphi$ when $\theta=22$ for different $\rho$. For $\rho=0$, from the horizontal view, the chaotic region is divided into several zones (Figure 7a). As $\rho$ increases, the chaotic zones gradually move to the left and shrink into almost one chaotic region, as shown in Figure $7 \mathrm{~b}-\mathrm{d}$. In fact, for $\rho=0.5$, the chaotic region also has a periodic motion with very narrow windows (in Figure 7e). As $\rho$ continues to increase, the chaotic region disappears and eventually the system becomes stable (Figure 7f). Moreover, the chaotic crisis still exists in the system when $\rho$ has some median values.

The variation of the bifurcation of flow of Route 1 with $\rho$ for different $\varphi$ is shown in Figure 8 . For $\varphi=0$ (Figure 8a), from the horizontal view, the chaotic region is similarly divided into several zones. As $\varphi$ increases, the chaotic zones move to the left and gradually shrink, as shown in Figure 8b-d. The narrow periodic windows disappears in some chaotic zones. For $\varphi=0.6$, the chaotic zones converge into one region (Figure 8e). The chaotic region disappears and eventually the system is stable as $\varphi$ continues to increase (Figure 8f). Meanwhile, the chaotic crisis also exists in the system when $\varphi$ takes some median values. As $\rho$ and $\varphi$ are both weight coefficients, and based on the preceding analysis, it is clear that these parameters have the same influence on the evolution of the system. 


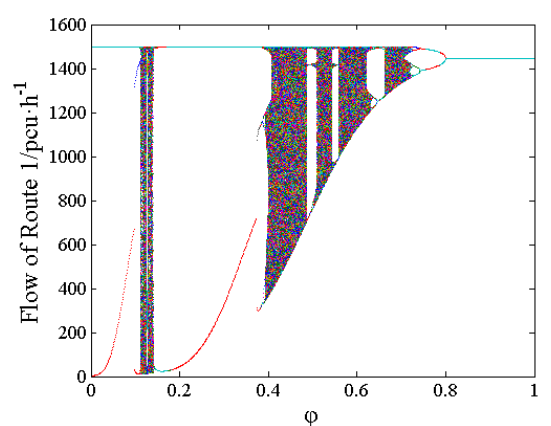

(a) $\rho=0$

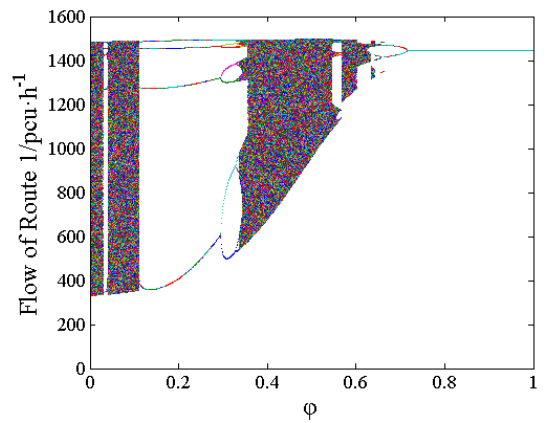

(c) $\rho=0.2$

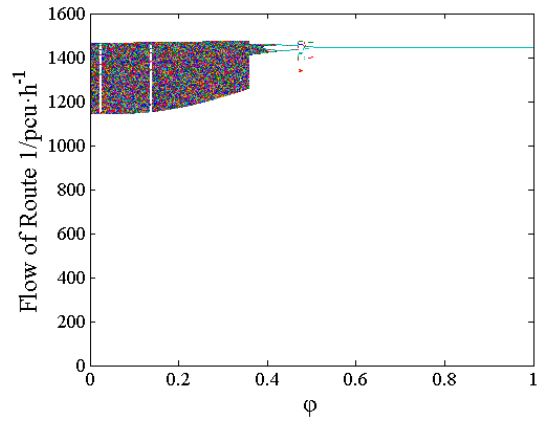

(e) $\rho=0.5$

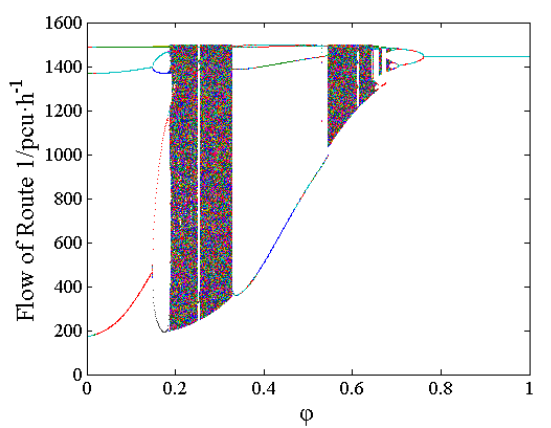

(b) $\rho=0.1$

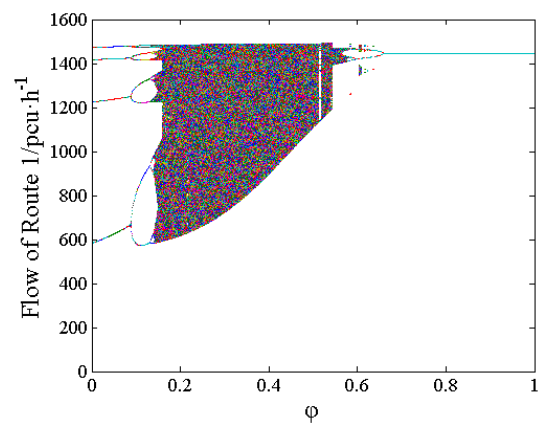

(d) $\rho=0.3$

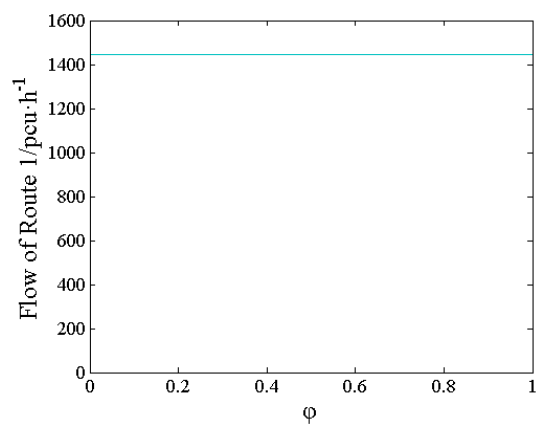

(f) $\rho=0.8$

Figure 7. Variation of flow bifurcation diagram when $\theta=22$ ( $\rho$ changes from 0 to 0.8 ).

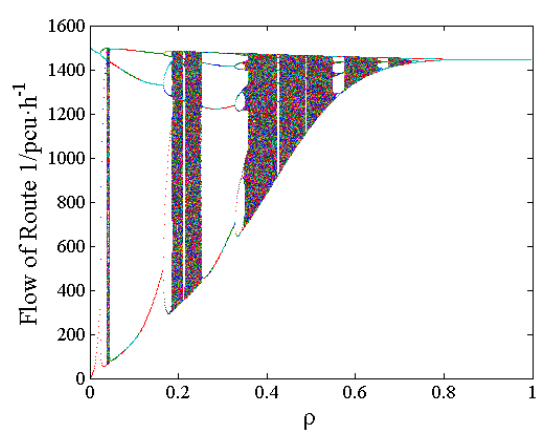

(a) $\varphi=0$

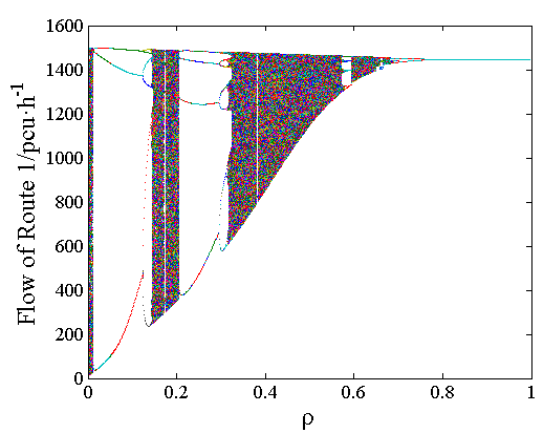

(b) $\varphi=0.1$

Figure 8. Cont. 


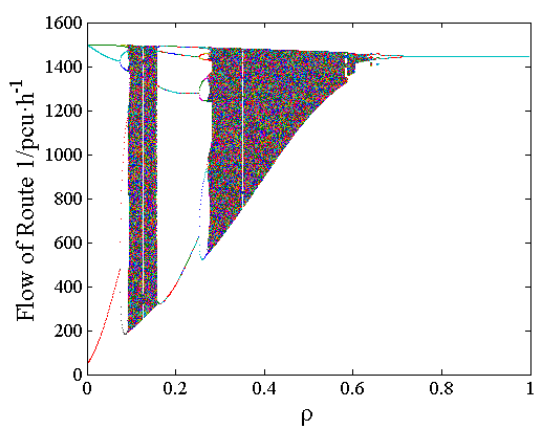

(c) $\varphi=0.2$

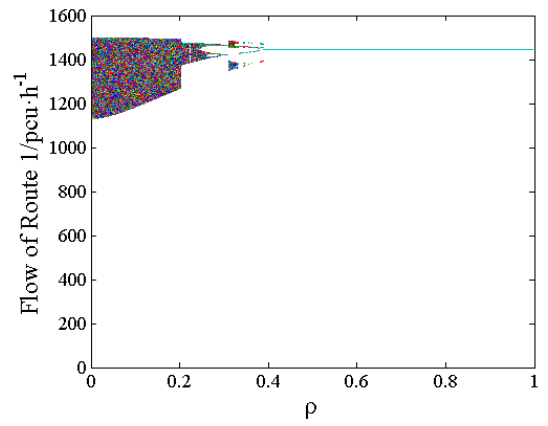

(e) $\varphi=0.6$

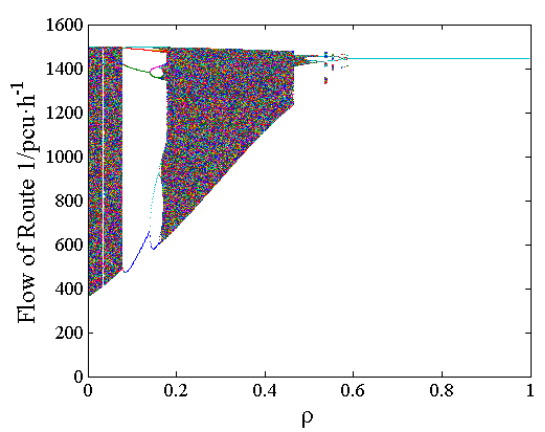

(d) $\varphi=0.4$

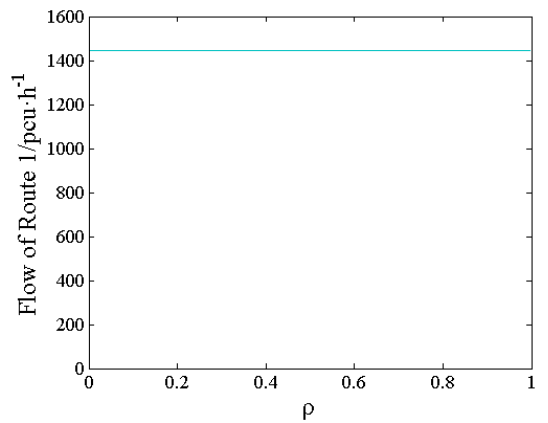

(f) $\varphi=0.8$

Figure 8. Variation of flow bifurcation diagram when $\theta=22$ ( $\varphi$ changes from 0 to 0.8 ).

Note that the parameters $\theta$ (travelers' sensitivity to travel cost) and $\varphi$ (traveler's reliance on the previous day's actual cost) have been analyzed in detail by Liu et al. and Li et al. [1-3]. However, they considered only the DTD updating of the travel cost and did not consider travelers' habituation in their evolutionary model. The traffic-flow evolutionary model presented here considers dual updating mechanism and considers travelers' habituation. Figure 7a corresponds to the case of $\rho=0$ (no travelers' habituation). As the evolutionary model of this paper is degenerated to the model formulated by Liu et al. [1], Figure 7a is similar to the corresponding figure in this reference. However, the travelers' habituation is involved for other values of the bifurcation parameter $(\rho \neq 0)$, so more characteristics are explored. Accordingly, this paper focuses on the influence of the parameter $\rho$ on the stability of system evolution.

When $\rho$ is different, the variation of the system's state with $\theta$ and $\varphi$ is shown in Figure 9. As noted, the stability, bifurcation, and chaos appear in the dynamic evolution of the system when $\rho$ is relatively small. As $\rho$ increases, the area of the stable region becomes larger, while the area of the unstable region becomes smaller, until the chaos and bifurcation disappear. Based on many numerical experiments, for $0.84 \leq \rho<1$, we found that the evolution of the network traffic flow is always stable regardless of the values of the parameters $\theta \in(0,+\infty)$ and $\varphi \in[0,1)$. 


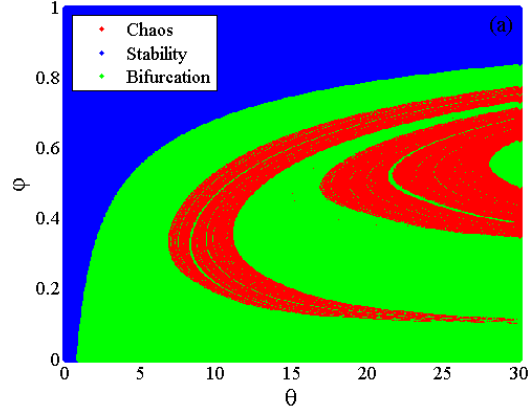

(a) $\rho=0$

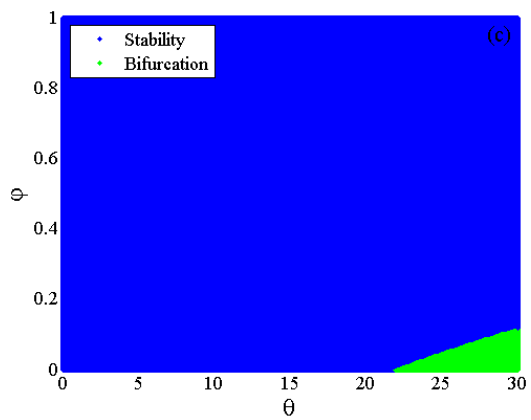

(c) $\rho=0.8$

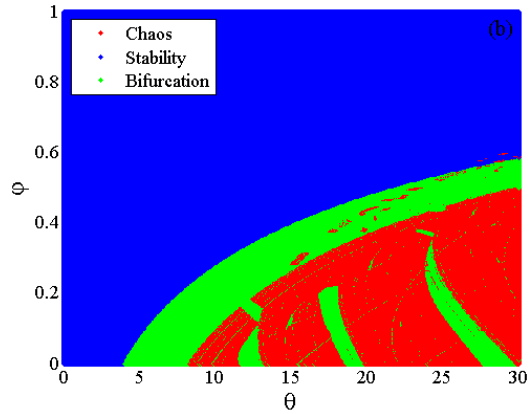

(b) $\rho=0.5$

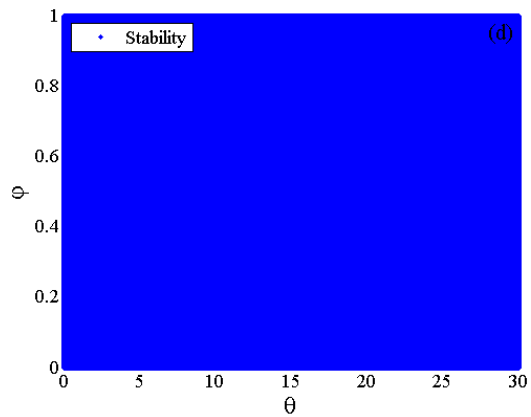

(d) $\rho=0.84$

Figure 9. System's state with $\theta$ and $\varphi$ for different $\rho$.

\section{Concluding Remarks}

This paper has presented a traffic flow evolutionary model under a dual updating mechanism that describes DTD traffic flow and travel cost dynamics. Considering travelers' learning and habituation, not only the travel cost, but also the traffic flow is updated in the evolution process. In the numerical experiments, the evolution characteristics of the dynamic system are analyzed by changing the parameters $\theta, \rho$, and $\varphi$, which are related to the characteristics of the travelers. Based on this study, the following comments are offered:

(1) Based on the developed Theorem 1, the equilibrium point of dynamic system is asymptotically stable under certain conditions. The results show that for $\theta \leq 0.923$, the equilibrium point is asymptotically stable regardless of the values of the parameters $\rho$ and $\varphi$. Bifurcation appears in the evolution of the system when $0.923<\theta \leq 4.402$. The results also show that the parameters $\rho$ and $\varphi$ have the same effect on the stability of the system.

(2) Chaos appears in the evolution of the system when $\theta>4$.402. As $\theta$ increases, the system will develop two kinds of chaotic crises, boundary crisis $(\theta>6.773)$ and interior crisis $(\theta>10.403)$. This indicates that when travelers are more sensitive to the route cost, the traffic flow will change suddenly with small variations in the parameters $\rho$ or $\varphi$, and the network system becomes extremely unstable. As $\theta$ continues to increase, taking $\theta=22$ for example, the chaotic region will be divided into several zones, indicating that the parameters $\rho$ and $\varphi$ have the same influence on the evolution of the system.

(3) The evolution of network traffic flow is always stable for $0.84 \leq \rho<1$, regardless of the values of the parameters $\theta \in(0,+\infty)$ and $\varphi \in[0,1)$. This result shows that when the proportion of travelers who do not change the route selected in the previous day is not less than $84 \%$, the evolution of the network traffic flows is always stable.

(4) The findings of this paper are applicable to only a two-route parallel network. For more complex traffic networks, the research method of this paper would provide a useful background for analyzing the network. The DTD dynamic evolution characteristics of the network traffic flows are analyzed by establishing a higher dimensional nonlinear dynamic model. However, 
the bifurcation and chaos phenomenon may be more complicated at present, such as the occurrence of hyperchaos. This research topic will be investigated in a future study. Additionally, more parameters can be analyzed. For example, the parameters of traffic network, such as free-flow link travel time and link capacity, can be discussed. If the number of lanes of one link increases, the link capacity will increase and may affect the results. In the future, the authors will conduct further research on this subject.

(5) As this line of research is still in the theoretical stage, it needs to be verified using actual data. First, we can verify the applicability of the model, the stability of the evolution of network traffic flow, the accuracy of bifurcation condition, and whether it will be chaotic in traffic using morning peak-hour flow of road network over time. Such data can be obtained from the traffic management departments of the cities. Second, if the chaos phenomenon of traffic flow is proved to exist using a large amount of field data, it will raise new research questions. For example, how chaos control can be carried out to make network traffic flow reach a stable state. This definitely will help traffic management departments to effectively manage and control road traffic. On the contrary, if the chaos of traffic flow is ignored, the traffic flow may be free today and congested tomorrow. Thus, it will cause the increase of fuel consumption of automobiles, and then air pollution will be aggravated. Therefore, the finding of chaos of network traffic-flow evolution and how to control chaos in the future is meaningful, because it is beneficial to sustainable transportation systems and the environment.

Author Contributions: Conceptualization, S.L.; Methodology, S.L.; Formal analysis, H.Y.; Investigation, H.Y. and S.L.; Writing—original draft preparation, H.Y.; Writing—review and editing, S.M.E.; Visualization, L.G. and Y.T.

Acknowledgments: The authors are grateful to the three anonymous reviewers for their thorough and most helpful comments. This research is financially supported by the National Natural Science Foundation of China (grant no. 51308126 and no. 71804026).

Conflicts of Interest: The authors declare no conflict of interest.

\section{References}

1. Liu, S.; Guan, H.; Yan, H. Chaotic behavior in the dynamical evolution of network traffic flow and its control. Acta Phys. Sin. 2012, 61, 58-67.

2. Liu, S.; Chen, W.; Chi, Q.; Yan, H. Day-to-day dynamical evolution of network traffic flow with elastic demand. Acta Phys. Sin. 2017, 66, 12-26.

3. Li, T.; Guan, H.; Liang, K. Day-to-Day dynamical evolution of network traffic flow under bounded rational view. Acta Phys. Sin. 2016, 65, 17-27.

4. Smith, M.J.; Watling, D.P. A route-swapping dynamical system and Lyapunov function for stochastic user equilibrium. Transp. Res. B 2016, 85, 132-141. [CrossRef]

5. Guo, R.; Yang, H.; Huang, H.; Tan, Z. Link-based day-to-day network traffic dynamics and equilibria. Transp. Res. B 2015, 71, 248-260. [CrossRef]

6. Cho, H.; Hwang, M.C. Day-to-day vehicular flow dynamics in intelligent transportation network. Math. Comput. Model. 2005, 41, 132-141. [CrossRef]

7. Mounce, R. Convergence in a continuous dynamic queueing model for traffic networks. Transp. Res. B 2006, 40, 779-791. [CrossRef]

8. Nakayama, S.; Kitamura, R.; Fujii, S. Drivers'route choice rules and network behavior: Do drivers become rational and homogeneous through leaning? Trans. Res. Rec. 2001, 1752, 62-68. [CrossRef]

9. Klügl, F.; Bazzan, A.L.C. Route decision behavior in a commuting scenario: Simple heuristics adaptation and effect of traffic forecast. Artif. Soc. Soc. Simul. 2004, 7. Available online: http://jasss.soc.surrey.ac.uk/7/1/1. html (accessed on 13 November 2018).

10. Selten, R.Y.; Chmura, T.; Pizt, T.; Kubec, S.; Schreckenberg, M. Commuters route choice behaviour. Game Econ. Behav. 2007, 58, 394-406. [CrossRef]

11. Kim, H.; Oh, J.S.; Jayakrishnan, R. Effects of user equilibrium assumption on network traffic pattern. KSCE J. Civ. Eng. 2009, 13, 117-127. [CrossRef] 
12. Zhang, C.; Liu, T.; Huang, H.; Chen, J. A cumulative prospect theory approach to commuters' day-to-day route-choice modeling with friends' travel information. Transp. Res. C 2018, 86, 527-548. [CrossRef]

13. Cantarella, G.E.; Cascetta, E. Dynamic processes and equilibrium in transportation networks: Towards a unifying theory. Transp. Sci. 1995, 29, 305-329. [CrossRef]

14. Zhang, X.; Jarrett, D.F. Chaos in a dynamic model of traffic flows in an origin-destination network. Chaos 1998, 8, 503-513. [CrossRef] [PubMed]

15. Watling, D.; Hazelton, M.L. The Dynamics and Equilibria of Day-to-Day Assignment Models. Netw. Spat. Econ. 2003, 3, 349-370. [CrossRef]

16. Guo, R.; Huang, H. Dynamical evolution processes of traffic flow and travel cost in urban transportation networks. Chin. Phys. B 2008, 17, 1698-1702.

17. Bie, J.; Lo, H.K. Stability and attraction domains of traffic equilibria in a day-to-day dynamical system formulation. Transp. Res. B 2010, 44, 90-107. [CrossRef]

18. He, X.; Guo, X.; Liu, H. A link-based day-to-day traffic assignment model. Transp. Res. B 2010, 44, 597-608. [CrossRef]

19. He, X.; Liu, H. Modeling the day-to-day traffic evolution process after an unexpected network disruption. Transp. Res. B 2012, 46, 50-71. [CrossRef]

20. Han, L.; Du, L. On a link-based day-to-day traffic assignment model. Transp. Res. B 2012, 46, 72-84. [CrossRef]

21. Guo, R.; Yang, H.; Huang, H. A discrete rational adjustment process of link flows in traffic networks. Transp. Res. C 2013, 34, 121-137. [CrossRef]

22. Rambha, T.; Boyles, S.D. Dynamic pricing in discrete time stochastic day-to-day route choice models. Transp. Res. B 2016, 92, 104-118. [CrossRef]

23. Ye, H.; Xiang, F.; Yang, H. Exploration of day-to-day route choice models by a virtual experiment. Transp. Res. Procedia 2017, 23, 679-699. [CrossRef]

24. Zhou, B.; Xu, M.; Meng, Q.; Huang, Z. A day-to-day route flow evolution process towards the mixed equilibria. Transp. Res. C 2017, 82, 210-228. [CrossRef]

25. Cantarella, G.E.; Watling, D.P. Modelling road traffic assignment as a day-to-day dynamic, deterministic process: A unified approach to discrete- and continuous-time models. Eur. J. Transp. Logist. 2016, 5, 69-98. [CrossRef]

26. Cantarella, G.E. Day-to-day dynamic models for Intelligent Transportation Systems design and appraisal. Transp. Res. C 2013, 29, 117-130. [CrossRef]

27. Zhao, X.; Orosz, G. Nonlinear day-to-day traffic dynamics with driver experience delay: Modeling, stability and bifurcation analysis. Physica D 2014, 275, 54-66. [CrossRef]

28. Ben-Akiva, M.; Lerman, S.R. Discrete Choice Analysis: Theory and Application to Travel Demand; The MIT Press: Cambridge, MA, USA, 1985.

29. Zhou, Y.; Cao, H.; Xiao, Y. Applications of Difference Equation; Science Press: Beijing, China, 2014. (In Chinese)

30. Liu, Z. Fundamentals and Applications of Chaotic Dynamics; Higher Education Press: Beijing, China, 2006. (In Chinese)

31. Lim, W.; Kim, S. Mechanism for boundary crises in quasiperiodically forced period-doubling systems. Phys. Lett. A 2005, 334, 160-168.

32. Jiang, H.; Li, T.; Zeng, X.; Zhang, L. Bifurcation analysis of the logistic map via two periodic impulsive forces. Chin. Phys. B 2014, 23, 112-118. [CrossRef]

(C) 2018 by the authors. Licensee MDPI, Basel, Switzerland. This article is an open access article distributed under the terms and conditions of the Creative Commons Attribution (CC BY) license (http://creativecommons.org/licenses/by/4.0/). 